\title{
Challenges In Managing Virtual Teams
}

\author{
Rudy Nydegger, Ph.D., Union Graduate College, USA
}

Liesl Nydegger, B.A., Claremont Graduate University, USA

\begin{abstract}
Many organizations are taking advantage of the opportunities to utilize new technologies to become more effective and efficient. One of the newer types of approaches to be used is the "virtual team." These are teams that are comprised of members who do not work at the same place or even at the same time. They may be spread across many time zones and may be located all over the world. These types of teams are made possible by advances in computer-mediated communication and software that allows people to work collaboratively on projects without being co-located or even working at the same time. Obviously, managing teams of this sort presents many, and sometimes unique, challenges. This paper addresses these issues, analyzes them, and offers suggestions for relevant management strategies.
\end{abstract}

Keywords: teams, virtual teams, computer-mediated communication, management strategies, team effectiveness

\section{INTRODUCTION}

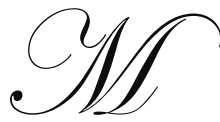

ost organizations today are trying to find new and innovative ways to be more competitive and efficient, and it is hardly surprising these often involve the use of new technologies. Without question, the emergence of new information and communication technologies has dramatically altered the way most organizations function. As organizations change the ways in which people communicate with one another, how they actually do their jobs changes as well. The workplace of the $21^{\text {st }}$ Century is a quite different place than that even a few years ago.

One of the changes that has been introduced in recent years is the use of "virtual teams." For many years we have seen organizations use conference calls, teleconferencing, and telecommuting as ways of helping people do their jobs more effectively, efficiently, and conveniently. However, virtual teams go beyond even these advances. It is now possible for teams that are not co-located (not in the same location) to work together on projects. In fact, people can work collaboratively on projects from different sites (all over the world if necessary), at different times, and even from different time zones. This is made possible by Computer-Mediated Communication (CMC) technology and by groupware and other software innovations that permit people to work together on programs, track the work and contributions, allow for communication between individuals and also with the entire group, and maintain a collaboratively established core document or project.

Keeping any team working together and communicating effectively is always a challenge, but with virtual teams these issues are far more of a concern. We know that it is necessary and important for people in organizations to keep in touch on a regular basis, and in most organizations today that is becoming increasingly difficult even with improved communication technologies. Even in "normal" co-located teams it is not unusual for team membership to be dynamic and change as needs dictate. Another trend that is common today is for teams to have members or consultants who function as a member of the team, but who are from outside of the parent organization. They are included in the team because they possess information, skills, or expertise that is essential for the team to have access to and which may not be available inside the organization. Further, it is common for workers to be involved in several teams at the same time and work on different projects simultaneously. Traditionally, work teams typically had specific projects with a beginning, a finite life span, and an end. Today it is more common for projects to be large, dynamic, and to evolve and change as circumstances dictate. Given these increased sources of complexity and complication for many (perhaps most) teams today, by adding the additional problems of having teams that are distributed (not co-located), working at different times, and perhaps working from different countries and time zones, and even with different native languages, one can see how quickly the levels of complexity dramatically 
increase. Another interesting aspect of organizations today is that many workers on different teams or in matrixtypes of organizations will have multiple-reporting relationships and will have different managers to report to for different projects on which they are working (Kimball, 1997).

\section{VIRTUAL TEAMS}

The use of virtual teams is a much more prevalent practice today, and it is being found in many different types of organizations and not just in the "high tech" arena. According to Duarte and Snyder (1999), virtual teams operate "... without the physical limitations of distance, time, and organizational boundaries. They use electronic collaboration technologies and other techniques to lower travel and facility costs, reduce project schedules, and improve decision-making time and communication (p. 4)." Gould (2006) simplifies this picture with the following equation:

Virtual teams=teams+electronic links+groupware

This equation points out that the three components of virtual teams include the people on the team, some form of CMC that will allow them to communicate interactively, and a software platform that will allow them to simultaneously work on a project at different times and from different places, and still track and integrate the work in a meaningful and efficient manner.

There are numerous changes and factors in organizational environments that make distributed work and virtual teams more attractive today. As organizations become more geographically dispersed and work with people in different locations and different time zones, these options become much more attractive. In addition, as organizations merge, acquire other organizations, are acquired by another organization, or subcontract work to different sites around the world, these types of teams are not only attractive but may be a necessity. Other realities like cost containment will also drive the decision to use these new forms of team management. Certainly, many organizations are finding that telecommuting and virtual work can dramatically reduce expenses and time and also allow for travel to be more strategically focused. In fact, the use of these types of teams can often reduce the timeto-market cycle which can be a significant benefit to customers (Nydegger, 2009).

There are many different types of teams that can be virtual. For example, they can, and often are, normal work teams, but they can also be executive teams and even teams that are made up of managers who work at different sites in the same organization. There is also a new type of team that is emerging in the virtual community and that is called the "community of practice team." This involves a group of people from different organizations who may be working on similar projects or problems that get together to support the work that they are all doing, even if it is for different companies (Kimball, 1997).

The opportunities for improved efficiency often drive many organizations to consider and implement virtual team work. In fact, Boiney (2001) found that over $2 / 3$ of Fortune 1000 companies are using self-managed and virtual teams. Cascio (2000) further argues that there are many good business reasons for using virtual teams:

$\begin{array}{ll}\text { - } & \text { Reduced real estate expense } \\ \text { - } & \text { Increased productivity } \\ \text { - } & \text { Higher profits } \\ \text { - } & \text { Access to global markets } \\ \text { - } & \text { Environmental benefits }\end{array}$

As attractive as these points are, the change to virtual types of teams is often a very difficult prospect for organizations. First, particularly in organizations with strong cultures, it may be quite alien to shift to this form of work and management. Further, many managers might find these types of changes threatening and may resist because they fear that this will reduce their power and impact in the organization. While the virtual teams can offer increased flexibility, responsiveness, and diversity of perspectives that differs from "normal and typical work groups," the differences between these teams and more traditional ones is sometimes a problem. 
Some of the differences between virtual teams and more traditional teams are fairly obvious; for example, they are usually geographically distributed, and sometimes rather extremely so. This, of course, usually means that the electronic communication between the members is the only tangible evidence that the group even exists. It is hardly surprising that these groups are often more informally structured and self-managed than are most traditional teams. What also happens is that there will be new secondary roles and differing status for group members, and it is also common that these new roles and status are completely independent of the roles and status that members have in their home organizations. The roles and status that members bring to the virtual team tend to depend primarily upon what they bring to the group - that is their roles and status in the virtual team depends very much upon the extent to which they bring something of value to the group. This seems to be even truer of virtual teams than traditional ones (Nydegger, 2009).

Some of the other differences that make virtual teams somewhat unique include the effects of anonymity and the lack of physicality (McKenna \& Green, 2002), which implies that issues like the maintenance of a clear group identity could be a problem (Finholt \& Sproull, 1990). Establishing and maintaining a clear sense of group identity is always an issue and often a problem in any regular group. However, in virtual teams there are far fewer cues to work with, less contact, restrictive contact, less personalized interactions, and fewer opportunities to develop and enhance the relationships that are vitally important to group and team success. Establishing and sustaining good group dynamics is a much more complex problem with virtual teams, but one that must be dealt with if the team is to succeed. We have known for years that a group cannot be effective if it does not have a stable normative structure. Without stable and functional norms any group will not, and in fact cannot, be effective. Similarly, roles need to emerge and develop as well. Since there are fewer social cues and more restricted communication in virtual teams, establishing consistent roles and expectations is not always an easy thing to do either. In virtual teams the information upon which role expectations are established is different than that in regular groups. Once again, this does not make the process less important, but it does make it more complicated.

One of the fundamental differences in virtual teams is communication. There is substantial evidence that one of the things that determines how effective a group or team will be is the quality of its communications (Nydegger, 2009). Some of the earlier work on CMC communication in groups suggests that it is not possible to employ the fuller range of communicative acts found in face-to-face $(\mathrm{FtF})$ groups in virtual teams. However, it is also emerging that as people get more experienced with the use of CMC communication they get more expert at imbuing their text messages with both task and social information (Walther, 1992). Observing how people use pictures, symbols, jargon and other shortcuts in their text-based communications demonstrates how much information one can add to the otherwise much leaner text-based communications. Certainly, these kinds of communications cannot possibly be as rich as FtF communications, but it is quite impressive how much more complete and complex text-based communications can be with more dimensions added in this way. It certainly appears that many younger people prefer texting to actually verbally talking to one another, and this generation will find working in virtual teams much more natural than their parents or older siblings.

It is generally accepted that communication is the fuel that allows groups to get their work done and to fulfill their goals. Obviously, communication is also fundamentally important in establishing the nature of the relationships among members of a team as well as the dynamics of the group or team itself (Nydegger, 2009). Since communication styles and patterns have to be different in virtual teams than in regular FtF groups, one might expect that interpersonal dynamics would differ as well. One study (Weisband \& Atwater, 1999) found that in normal FtF groups individual liking between group members was largely based on non-rational or non-task bases of attraction, although in virtual teams people seem to like those who contribute most to the group's performance. This seems entirely understandable, but it also indicates that as organizations begin using more virtual groups, they will need to pay attention to how communication is different and to facilitate the best possible communication under the circumstances. In fact, as we will see later in this paper, training is essential if we expect virtual groups and teams to be as effective as they will need to be.

According to Bjørn and Ngwenyama (2009), the risk of communication breakdowns in virtual teams increases due to cultural and organizational differences that are the result of being geographically distributed. They feel that these breakdowns are caused by general misunderstandings and the absence of "shared meaning" between the participants. In fact, numerous studies have shown that effective communication in the virtual environment is 
dependent upon the "time-consuming process" of establishing a common ground (Bjørn \& Hertzum, 2006; Malhotra \& Majchrzak, 2004). Others have pointed out that establishing a common ground is basically a process of creating a "shared meaning context," and that failure to provide this context often results in serious breakdowns in collaborative efforts (Cramton, 2001). Similarly, Malhotra and Majchrzak (2004) have pointed out that knowledge sharing is another fundamental feature of collaboration that is closely related to the establishment of a shared meaning context. They argue that in the process of building consensus, knowledge is shared and vice versa. In other words, knowledge sharing facilitates better consensus building, and in a cohesive group with a history of consensus building, knowledge sharing is usually better as well.

Another vitally important aspect of team effectiveness is trust, and because of the lack of important social and non-verbal cues in virtual teams, this becomes even a bigger issue. Box, et al. (2002) demonstrated that as with other relational dynamics in groups, trust tends to diminish when there are fewer visual and vocal cues as in CMC groups. Since the establishment of trust depends in large part on the exchange of information (Jarvenpaa \& Leidner (1998), helping virtual teams find ways to enrich their communication is very important. As we know, trust is important among group members so that they will enjoy their jobs and feel a part of the team, and in one study trust was found to be positively related to group performance (Cascio, 2000).

Very much related to the issue of trust is cohesiveness, which is the extent to which members feel connected to and a part of the group. Goodman, et al. (1987) established that cohesiveness is very important in determining how effective a group is, and yet much of the work on CMC teams shows that these types of groups show lower cohesiveness and feel more loosely connected to their groups (Lea \& Spears, 1991). There is no question that if virtual teams are to be effective they must feel a sense of cohesion and connectedness. These factors obviously depend upon members trusting and liking one another and these in turn depend on the quality, type, and amount of communication they have.

When these factors are established, CMC groups can and do run very effectively and the resulting outcomes can be quite rewarding. For example, in high-functioning CMC groups a number of things have been established:

- $\quad$ Greater liking for and acceptance by other group members

- $\quad$ Negating the effects of social anxiety

- $\quad$ Decreased feelings of isolation and loneliness

- $\quad$ Increasing one's social network

- $\quad$ Coming together and feeling part of the group (McKenna, 2008)

On the other hand, it is also true that like any group, virtual teams can become dysfunctional because of a variety of factors. It is clear that problems with communication and lack of trust can disrupt any group including virtual ones. Sometimes, virtual teams will run into trouble because of misunderstandings or misapprehensions regarding the nature and quality of virtual groups and how they function. It is not uncommon for people to have beliefs and feelings about things that they don't fully understand, and some of the myths that have grown up around virtual teams clearly demonstrate this. For example, some (perhaps many) people entertain one or more of the following mistaken beliefs about virtual teams:

- Virtual teams don't need supervision — since members usually work alone they don't need much from leaders, managers, or supervisors.

- I It is very easy for members of virtual teams to avoid work and to "hide." Virtual team members cannot be trusted to work without being monitored closely.

- You can't manage from a distance, and thus managers can't really have much of an impact on the performance of virtual teams.

- $\quad$ Because people don't really work very closely together in virtual teams, trust isn't as big an issue, and you can't really establish much trust in these types of groups anyway.

- $\quad$ Virtual teams don't need FtF contact and they usually prefer just interacting through the CMC technology (Nydegger, 2009). 
Of course, all of these statements are myths and none of them are true. However, as we have learned by studying self-fulfilling prophecies, if one believes something will happen it often does. W.I. Thomas (1923) stated this very clearly when he pointed out that, "Situations people define as real are real in their consequences." That is why we need education and training to help people understand the realities and peculiarities of virtual teams and not get mislead by fallacious and often harmful myths.

One thing that is vitally important for managers, organizations, and virtual team members to keep in mind, it that for the important differences between virtual and "normal" teams, they are still teams, and have many of the challenges and strengths of any team. For example, Hertel, et al. (2005) point out the lifecycle of virtual teams as manifesting five distinct phases. These are:

- $\quad$ Preparation: this is where the mission statement, team composition and membership, the fundamental task design, the reward systems, the relevant technology, and the organizational integration are established.

- $\quad$ Launch: here is where there is an initial workshop or introductory session where people meet one another, goals are clarified, and the intra-team rules are established.

- $\quad$ Performance Management: in this phase leadership patterns are established, regulation of communication is developed, motivational and emotional issues are relevant, and knowledge management methods are introduced and agreed upon.

- $\quad$ Team Development: now the team will assess its needs and deficits, utilize additional individual or team training, and evaluate the effectiveness of the training.

- Disbanding: in the final phase, the achievements are recognized and the team members are re-integrated into their home organizations.

Clearly, these stages are reflective of the unique needs and experiences of the virtual team, but it is also very interesting to note that they are very similar to those group development stages first mentioned by Tuckman in 1965 and later amended in 1970. Tuckman's stages, Forming, Storming, Norming, Performing, and Adjourning, describe patterns found in almost all groups (including virtual teams), and this clearly demonstrates how fundamentally "group-like" virtual teams are, but also how we can utilize much of the earlier work on teams to help us understand and manage virtual teams more effectively. Hertel and his colleague's (2005) work is a very useful upgrade of the original model, and is particularly good at showing some of the unique elements of virtual teams that set them apart from other types of teams.

However, we must also remember that virtual teams are similar to regular teams in many ways, but the differences are critically important to recognize and deal with if teams are to be successful. One very powerful reminder of this fact comes from a study by Dubé and Robey (2008). One of the project managers in their study reported that he had tried to manage his virtual team just like a normal team, and his denial of the true differences between these different types of teams resulted in a project that was very late which upset the client and created a major failure for the company. As they point out, denial does not prepare virtual teams to survive their challenges (Dubé \& Robey, 2008).

\section{ADVANTAGES AND DISADVANTAGES OF VIRTUAL TEAMS}

It should be obvious that there are some qualities and processes in virtual teams that make them different from normal FtF groups. Some differences emerge because of relational dynamics, discretionary and asynchronous participation, free-riding, difficulty integrating information and other information-processing aspects of virtual work that make virtual groups especially difficult to manage (Smith \& Vanacek, 1990). Interestingly, there are some aspects of virtual groups that make them uniquely advantageous. For example, they allow for efficient communication with people all over the world at any time. Some people even find that there is an intimacy among members in a virtual group that is very special, and perhaps this stems from interacting with one another in more comfortable settings and at particularly convenient times (Nydegger, 2009).

There are emerging some clear patterns that suggest that virtual teams have some very significant advantages, and that they can be powerful and appropriate ways to get work done. Since communication in CMC virtual teams is very fast and efficient it is easier to get information where it needs to be, and it is also easier to 
spread "best practices" among workers. Further, it becomes far simpler to connect "islands of knowledge" into selforganizing, knowledge-sharing networks of professional communities and fostering cross-functional and crossdivisional collaboration. It also becomes simpler to initiate and contribute to projects and to communicate across organizational boundaries (Kimball, 1997). As we have more experience with virtual teams it is also clear that there are very few teams that are $100 \%$ virtual, and they tend to take on some of the characteristics and qualities of normal teams, but in a virtual setting. Most importantly, evidence and experience is showing that people can be trusted to work in virtual settings and that virtual teams tend to get their work done (Gould, 1997). Other advantages of virtual teams include:

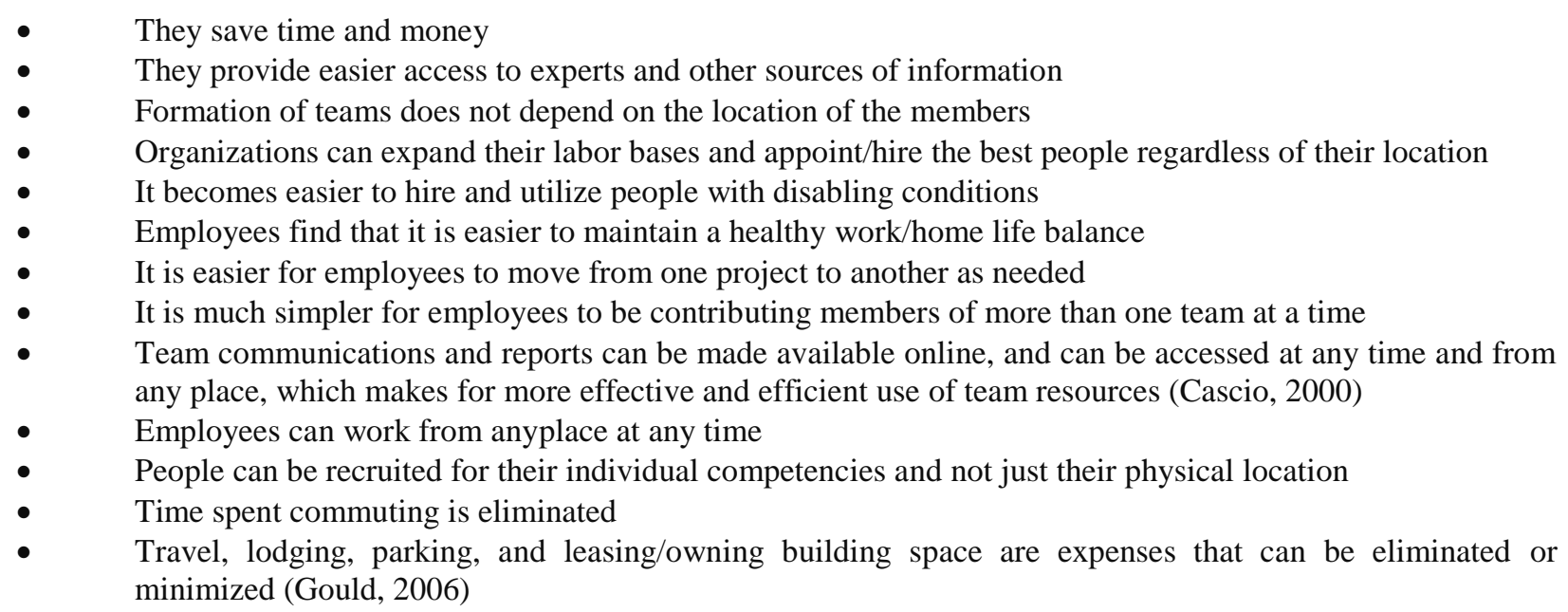

Employers cannot underestimate some of the cost savings of taking advantage of distributed and virtual work. One study that was done by the U.S. government demonstrated that if 20,000 federal employees could telecommute one day per week they would save over two million commuting miles, 102,000 gallons of gasoline, and 81,600 pounds of carbon dioxide emissions each week (Cascio, 2000). Obviously, savings of this magnitude are substantial and very compelling, and clearly address issues that many organizations must confront. Organizations from the largest to the very humble and modest must aggressively pursue any opportunity to improve effectiveness, efficiency, and cost effectiveness. For some, this may involve the used of distributed work and virtual teams.

Without question, virtual groups and distributed work have many significant advantages, but there are some important drawbacks and disadvantages as well. Interestingly, and not surprisingly, there are often mistaken opinions and perceptions of the causes of problems in virtual teams which sometimes makes it difficult to deal with the actual causes of the problems. For example, sub-par performance in virtual teams is frequently blamed on the technology first, but in reality it is more frequently individuals or social factors that are responsible for the performance decrements (Kimball, 1997). Another study found that both men and women report that the "real" problems in performance difficulties in virtual teams come more from poor sharing of information, unclear or inappropriate expectations, and unclear lines of accountability or control than from technology problems (Boiney, 2001). However, that is not to say that the technology cannot be a problem because it obviously can be. However, it is important to accurately attribute the causal locus of the difficulties and not simply scapegoat the technology because it cannot defend itself. When technology is the problem it should be addressed, but when it is not that should be addressed as well.

There are other problems associated with the use of virtual teams. For example, the cost of setting up and maintaining the expensive hardware and software necessary to support virtual teams is not insignificant and might even be prohibitive for some organizations. A careful cost/benefit analysis must be done before a decision can be made to implement a costly system that requires substantial attention to maintain. The sophisticated communication and information technology infrastructure necessary to manage a virtual team must be considered within the context of the communication environment, MIS, organizational culture, management strategies, financial constraints, human resource capabilities, and physical environment within which the virtual teams must function. As cost 
effective as distributed and virtual work might be it must also be balanced off against the savings to be gained by having the work done in one location without the expensive support needed by virtual teams. Some of the softer costs must also be considered as well. For example, cultural issues, feelings of isolation, and a lack of trust related to more limited interaction with managers and supervisors are all things that must be considered and dealt with in the virtual workplace (Cascio, 2000).

It does appear that the major difficulties with virtual teams has to do with the fact that being distributed and not co-located may lead to difficulties with things like relational communication and trust, but also with outcome problems like decreased productivity and work quality. Relational communication has to do with the regard that members have for one another and how they express that regard. Since distributed teams lack some of the richness of FtF teams, and particularly in the area of paraverbal and non-verbal cues, it is true that these non-co-located teams are at a disadvantage with respect to relational communication, and this too will negatively impact things like trust (Walther, et al., 2005). Some, in fact, have asserted that trust cannot be maintained in virtual teams (Handy, 1995). While we do not agree with this statement, we do believe that trust is established and maintained differently in virtual teams, and this is probably more difficult than it is in FtF teams. Virtual team members' perceptions of mutual trust between themselves and their manager and organizational peers played an important role in the workers' adjustment to the work in the virtual team. As in other teams, the relationship between the team members and the manager is critical to the success of the virtual team. Trust is often a fragile aspect of virtual teams because of the limited opportunities for relationship-building exchanges, and virtual team members often have lower perceptions of managerial support and have significantly less trust than their "non-virtual" colleagues in regular teams. This reduced trust will interfere with work performance, increases turnover intentions, results in lower employee support of management, and hinders employees' adjustment to the virtual work environment (Merriman, et al., 2007). In some teams the virtual team appears to be self-managed, but in reality they only solve problems but are not allowed to make decisions, and this lack of authority will lead to the team members learning to mistrust their managers even more (Oakley, 1998). If managers want and expect the virtual teams to be fully engaged and productive they must give them authority as well as responsibility, and as Oakley (1998) points out that when managers try to use traditional supervisory monitoring and oversight methods, they are undermining the effectiveness of the team until control is replaced by trust.

There are many factors that lead to virtual teams being more challenging to manage than traditional teams. Different types of organizational settings and environments, different cultures and norms, in the various sites and different time zones are but some of the issues with which the manager and employees must deal. However, the main problem has to be the lack of physical interaction and the absence (or near absence) of non-verbal cues (Nydegger, 2009). Merriman et al. (2007) suggested that virtual employees can often feel unimportant such as "being out of sight, out of mind." Clearly, this lack of richness of communication makes the subtleties and nuances of normal social interaction much more difficult to sort out. This is likely to be an even bigger problem in highcontext communication cultures like China, Japan, and many of the Middle Eastern and South American countries. In high context cultures the settings and context of communication as well as the non-verbal and social cues are fundamental to good and accurate communication (Nydegger, 2009).

One problem with virtual teams that has been recognized but is often ignored or neglected is the resistance that many managers have to the use of virtual teams. Some managers feel that virtual teams are a threat to their identities because they may feel that their role, authority, and responsibilities are unclear in this new setting. They may also feel that there is an issue related to status and esteem, and many may feel that they have less control over the work for which they are responsible (Wiessenfeld, et al., 1999). While it is understandable as to how and why some managers may feel this way, it is vitally important than managers be on board with these types of programs. To accomplish this, managers must be adequately informed and trained to deal with the new situations. They must understand and appreciate the advantages and challenges of the changes to a virtual workplace, and they need to accept and be committed to their new roles. They will see that although their roles might change, the new roles are not less important, and in fact there will be new opportunities for managers to excel and to make their mark (Nydegger, 2009). If the managers do not embrace and get excited about their new roles and responsibilities it is not likely that the teams will succeed. Therefore, care must be taken to inform, educate, train, listen to, support, and empathize with the managers as they start to work in and with an entirely new way of organizing and utilizing teams. 
Another interesting observation on virtual teams comes from the work of Dubé and Robey (2008) who point out that virtual teams are filled with paradoxes that must be recognized and dealt with if teams are to be effective. To deny or avoid these paradoxes clearly dooms the team to sub-par performance. Some of the paradoxes that they discuss include:

- $\quad$ Virtual teamwork is flexible, yet must be supported by structural mechanisms that help coordinate team efforts - the work is by necessity and nature complex and requires flexibility to accomplish the goals, however without some structure and "grounding" the work cannot and will not be effectively coordinated.

- Teamwork implies interdependence among members in accomplishing their common goals, and yet the work ultimately must be subdivided and done by individuals - this is, of course, true of almost any team, but it is particularly clear in virtual teams because of the distributed nature of the work and working conditions; they cannot do the job without working together, but each person must be accountable for their share of the work or it will not get done, or at least will not get done as well as it needs to be.

- Trust is absolutely necessary for virtual teams to be effective, and yet mistrust is also an opportunity for trustworthiness to be achieved - for trust to be established in any relationship there must be willingness to trust and an intention and desire for the other party display trustworthiness.

- Virtual teams are geographically distributed and workers often work from different places and at different times, and yet virtual teams do require the physical presence of other members - while the work must get done in the virtual setting, it is important for physical relationships to become the basis of the virtual ones, and both are important.

- Virtual teams are task-oriented because of the nature of their work, but they depend on social interactions to actually complete their tasks - we know that being distributed necessitates focusing on tasks, but these cannot be completed without working together.

As important and advantageous as virtual teams might be they are not the "answer" for all teams or organizations. Clearly, as the sophistication of the CMC technology grows and the hardware and software options become more advanced we will see increased interest and use of virtual and distributed teams and they will become increasingly common and valuable. However, this is not to say that they will ever totally replace the traditional FtF teams because there are things for which the FtF team will be essential. A meta-analytic study comparing the effectiveness of $\mathrm{FtF}$ and virtual teams was inconclusive as to which was superior (McLeod, 1992), and although this study is more than a decade old, its findings have appeared to have held up. It is not likely that this result suggests that there is no difference in the effectiveness of these two types of teams, and it is more likely that this result suggests that although there is no overall superiority of one type of team over the other, this is probably due to the fact that each type of team is superior in specific situations, but not in all. If this is true, and we think it is, it must be established in the professional literature. What an enormous contribution if researchers can start identifying and clarifying the types of situations that will be best suited for each specific type of group or problem. While this has yet to be done it is a very logical and promising avenue for new research to pursue.

\section{SOME TIPS FOR MANAGING VIRTUAL TEAMS}

When considering the management of virtual teams it is tempting to first think about the technology, and while this is important it must be remembered that virtual teams like any other team is first and foremost a social system. Therefore, regardless of the communications technology or the hardware and software that the group uses to do their work, the success of the team will still depend upon the quality of the information available, how it is communicated and used, and how the team members work together with this information to complete their tasks. No group is better than the quality of their information and their team members. What one hopes for in a group is that the performance will reflect a synergy that emerges from the group as a fully functioning social work unit. This, however, does not emerge automatically and takes communication, trust, sharing information, and training, working together collaboratively, and surpassing the performance of the individuals alone.

Many organizations today rely more on team-based management structures, but in all honesty and in our experience, very few organizations do this well. First, although many organizations talk about empowering teams, they rarely give teams the authority they need to fulfill their responsibilities, and this is a common problem in many organizations. With virtual teams this is even a bigger problem because the traditional role of manager or supervisor 
will depend upon them giving the team considerable authority for their work because if that does not happen and the manager has to be involved in every element and decision, they then become the bottleneck that will prevent the group from functioning optimally. Therefore, it is essential that the virtual team has adequate resources and the authority to use them as they need to for the purpose of fulfilling their goals. Secondly, it is also our experience that most organizations still design work around individual jobs rather than teams. Very often, managers still think of teams as a collection of jobs rather than a group of interdependent, collaborative colleagues working together on a common task or project.

Another factor that can be very important in virtual teams is empowerment. This could be extremely beneficial to virtual teams if used appropriately. Dubé and Robey (2008) suggest that since monitoring member activities is often difficult in virtual teams, empowerment could improve performance. If managers of virtual teams coordinated team activities rather than directed them, they could be more effective. An empowered and empowering manager will have characteristics of a democratic power orientation, low levels of involvement, self-regulates, is a boundary spanner, and helps team members in any way necessary (Oakley, 1998).

Many of the functions that are necessary for managers in the virtual team environment are somewhat different that one might find in typical $\mathrm{FtF}$ teams, but as mentioned above these are certainly no less important. For example, managers must facilitate all members feeling a part of the team. Merriman et al. (2007) suggest that managers should encourage trust throughout the team related to business concerns rather than personal relationships, which may facilitate how quickly team members begin to trust each other. Further, they must also engage in the practices of the group both as an observer and a participant so that they enhance their own knowledge and experience. This type of engagement is inseparable from empowerment, and when managers fail to perform as they need to it is often because of exclusion from the group process (Kimball, 1997). Managers need to be part of the process so that they know what is going on and are able to support and facilitate better group functioning. Further, by being part of the process and gaining information and expertise, the manager also gains credibility with the group members and they are more likely to listen to and seek advice/support from that manager.

For managers to effectively manage virtual teams there are many things that they must do to provide a context and the support necessary for healthy and productive virtual teams to flourish. Kimball (1997) suggests some things that managers can do to help the virtual team succeed:

- $\quad$ Purpose: teams need to feel a unified sense of purpose; schedule more frequent and explicit check-ins since there is a lack of FtF meetings.

- $\quad$ Roles: since role ambiguity and confusion are usually related to poor performance, it is necessary for managers to make sure each member has a clearly defined role that is understood and accepted by all group members; this role may be appointed or emergent, but it must be explicit.

- $\quad$ Culture: the prevailing culture of the organization establishes a context of which each team must be a part. Technology must be used to facilitate the virtual team being a part of the culture, but the dynamic relationship must also be recognized and dealt with; that is, the team is affected by the culture but will affect the culture as well.

- Conversation: managers must support and encourage conversation, discourse, questioning, etc. among group members; the technology cannot only be used for data exchange and storage.

- $\quad$ Feedback: spend time using technology to improve the quality of communication among all of the members of the team; this is the primary way to produce frequent, timely, and helpful feedback. This is likely to be most important to the older members who may be less experienced in using CMC.

- $\quad$ Pace: managers must try to facilitate a reasonable pace at which work is done. It is likely that some people will use the shared environment at a higher rate than others. However, you do not want some members feeling like they are being left behind. In an asynchronous environment keeping people on board, participating, and feeling part of the team is not easy, but it is essential.

- $\quad$ Entry and re-entry: when bringing new members on board in a virtual team it is rather like jumping into a fast flowing river and trying to catch those who jumped in before you. Managers must try to get new members (or returning members) updated and integrated into the process as quickly and efficiently as possible. 
- Weaving: this is a networking term that describes the process of summarizing and synthesizing multiple responses in virtual teams. This means you need to tell people where they have been, where they are, and where they might want to go next.

- $\quad$ Participation: Managers need to be aware of the cues that are unique to virtual teams that indicate when people are engaged and participating, and when they are not. In FtF teams there are more non-verbal cues to assess levels of participation more easily, but in virtual teams there are also cues, but managers must be able to recognize them. For example, emoticons (textual representation of mood or emotional state) and other "paramessages" will give richness to a "lean" form of communication, but they are only functional if the people who see them understand what they mean.

- Flow: the manager must be aware of what is happening (or not happening) in the group. Like any group, virtual teams will be very active as some point and less active at others. While this is normal, the manager must monitor this and be on top of the process so that the team is working together effectively and efficiently.

Kimball (1997) also recommends that before an organization starts to implement virtual teams a number of things should happen. For example, the first thing that should happen is that the process for managing and developing the teams must be designed, defined, piloted, tested and refined before the teams are fully brought on line. Rather than spending a lot of time chasing mistakes and unforeseen problems, the groundwork must be laid carefully and completely. In the excitement to get things started quickly, many organizations leap at virtual teams before the foundation is laid, and invariably problems emerge and the teams have considerable difficulty being effective. The next thing that must happen is training. We often assume that people do not need to be trained to work in teams and virtual teams are just another type of team. Experience dictates that training is helpful in any team, but in virtual teams it is essential — you are asking people to work collaboratively in ways that are entirely new and unpracticed. Training will save time and trouble and will actually get teams online and productive much faster - time spent training will save even more time and money. Another important factor is that new virtual teams will be part of an existing culture that did not initially include them. The culture will have to be modified to reflect the use of a new and different type of team model. Next, the reward system must be altered to reflect the new ways that work is performed and evaluated. For many organizations the use of team-based reward systems is new and often quite alien. Evaluating both individual and team performance is very important if these types of teams are to be optimally effective. Finally, new management, measurement, and control systems must be designed and integrated into the processes as they relate to the new types of groups and become part of the organization as a whole. following:

Some specific steps that management can take when instituting a new virtual team system include the

- $\quad$ Start by modifying the culture, and then support the change by using new technology.

- $\quad$ Change and broaden the reward system to evaluate employee's teamwork, their ability to help the team meet its goals, and their ability to share information.

- $\quad$ Support and encourage ground-up, grassroots efforts.

- $\quad$ Ensure that new software fits the new processes, is installed properly, and that the team members are adequately trained in its use.

- If at all possible, when starting new virtual teams try to begin the early collaborative work with FtF meetings.

- $\quad$ Rely on role modeling as the main way to spread the use of the relevant groupware.

- $\quad$ Use relevant training programs for teaching skills and the use of the software (Nydegger, 2009).

It is clear that using virtual teams is a major commitment and one that is worth making sure that it will be effective and functional in a timely manner. To do this the right way will depend on getting the right people on board, spending money on the appropriate hardware and software, attracting and training the right people, and designing a management structure and reward system that will guide the work and recognize and reward the contributors. Too often, organizations will try to do too much too soon without adequate support and then blame the technology or the teams for not being successful when they did not have much of a chance to begin with. For 
something like this to work there needs to be commitment and support from the top down, and the messages need to be consistent and supportive. Very often, major changes like virtual teams are met with resistance and organizational politics which are understandable but terribly disruptive and potentially problematic.

Gould (1997) points out that the most common complaint made by members of virtual teams is communication - not enough, distortions, unclear messages, etc. He also recommends that at least the first meeting of the team be FtF, but also suggests that occasional FtF meetings will improve communication and reduce some of the problems that result from the challenges of trying to communicate using the new CMC technology. He also suggests that some of the difficulties with dysfunctional conflict and delays can be improved with a code of conduct that all members are made aware of from the very beginning and are expected to follow at all times. Further, to improve communication, team members should also be encouraged to enrich their text-only messages with non-textbased supplemental information that will make their messages richer and more personal (e.g., "emoticons" or other non-text based ways of expressing feelings and other socially relevant information). This will improve the quality of the communication but will also lead to more and better trust among the team members.

Bjørn \& Ngwenyama (2009) point out another element related to communication that is very important in the virtual group environment - translucence. This refers to groupware design features that permit invisible social cues to become visible making it easier for members to understand the subtleties and deeper meanings of the work they are doing together. Translucence is a vitally important element in collaborative technologies and should be made available in a "low-effort, seamless way" that does not interfere with the primary tasks (Ebling, et al., 2002). With the relative absence of typical non-verbal and paraverbal cues in the virtual team, translucency in the technology realm makes true collaboration much easier and more efficient.

Starting a virtual team is not always easy to do. For example, you must lay the appropriate groundwork and provide the right kinds of support, but you must also get things moving in a timely fashion. Some basic rules that can help lead to success are:

- $\quad$ Start right away—do not unnecessarily slow things down.

- $\quad$ Make sure to communicate frequently and to all members of the team.

- $\quad$ Engage in the organizing and performance of substantive work simultaneously.

- $\quad$ Acknowledge clearly that you have received and read one another's messages.

- $\quad$ Be explicit about your activities - tell people what you are doing and what you are thinking.

- $\quad$ Establish and respect deadlines - make them and stick to them (Walther, et al., 2005).

There is research and experience that supports the idea that when these six rules are followed the virtual teams are more effective, but also that team members were more likely to report being satisfied with their experience on the team and with their interactions with other team members (Walther, et al., 2005).

Experience also suggests that teams do better when encouraged to set their own goals for both performance and behavior, and this reduces confusion, ambiguity, and conflict. Using goal setting and task design can also help with interdependence and compensate for feelings of disconnectedness (Hertel et al., 2007). Clearly, goals must first reflect the outcomes sought by the organization, but the personal and interpersonal goals of the members must be respected as well-at least as long as they are functional and not disruptive. Leaders and members must also be aware of the importance of setting the right tone for the group. An atmosphere of openness and honesty is one of the most important factors in setting the stage for a group being successful. However, conflict will and must arise in any group, and this is helpful too. To keep the conflict functional and productive, leaders must encourage members to voice differences of opinion and disagreements, but also instruct them as to how these issues will be addressed and managed. Managers must do everything possible to ensure that communication is open, frequent, accurate, and as rich as the situation and circumstances will support. Similarly, leaders must encourage and support frequent, honest, and helpful feedback, as well as frequent formal and informal communication sessions to make sure that all members are involved and productive, and to deal with any issues that might be lurking below the surface. By clearly communicating expectations, and making sure that the rewards support the accomplishment of those expectations, managers can have a substantial impact on the performance of the teams and the quality of their products. 
Managers today will need to have a new mindset about work, jobs, teams and performance. Most managers think in terms of specific jobs with identifiable outputs. Today, however, much work is done in teams and this requires a different approach to management. Adding the virtual component to teams requires an even more radical change of mindset. Mangers must now think in terms of setting up the most important type of work unit for the job at hand and the technology available. Getting the right people to work together on a specific project may mean that the manager may have to go to the virtual team model to accomplish his/her goals. Virtual teams are not always the answer, but FtF teams are not either. Fitting the type of team to the task at hand will be a new management skill that will have to be mastered in organizations today (Nydegger, 2009).

Some other changes in managerial perspective will also be needed. For example, all teams are and will be people-oriented - they have to be. However, the ways in which managers relate to the team members will be somewhat different in virtual teams. They will need to trust team members to work and get their jobs done without direct supervision, and while this may be a leap of faith for some, it is necessary. If the manager feels that they have to be involved and on top of everything that happens, this is when they become the bureaucratic bottle-neck that is more of an impediment than a help. Having an open and positive attitude that focuses on opportunities and solutions rather than on blaming and excuses is important as is the avoidance of continually finding reasons why the virtual team is a mistake and should be changed. Effective leaders in any group should focus on results rather than on processes, and by using effective communication, both formally and informally, they will give their groups a significant advantage in the accomplishment of their goals. Finally, good managers in any setting know how and when to delegate, and how to follow-up with feedback and support to make certain that the work is being performed as it should (Cascio, 1999).

For virtual teams to succeed, commitment for virtual work must start at the top of the organization and touch and include all members and all levels of the organization. It is a commitment that will and should touch everyone whether they are on the team or not. This type of change will impact the culture of the whole organization and will affect the way work is conceptualized, designed, supervised, and recognized. The most important aspect of the virtual team - any team in fact-is communication. Team members must be able to be in touch with one another through CMC, telephone, and occasional FtF meetings. Likewise, all members must be able to communicate with their supervisors and managers in a timely manner as well. Good communication is a necessary but not sufficient condition for team success; there are many other things that must happen as well. The team members must be aware of and share the goals for the group, and see how those goals relate to the mission and vision of the organization. Since experience has clearly demonstrated that diversity is a very positive factor in group effectiveness, putting together appropriately diverse teams, managing them effectively, and helping them learn how to work together productively is a major management challenge, but one that will yield positive results. However, the more diverse the team the more challenging the communication and the more difficult they are to manage. Helping team members get to know each other on a personal level is clearly an advantage to helping the team work together effectively, and once again, occasional FtF meetings can help this happen. Probably the most important element of the effective virtual team is the fundamental notion that trust and respect are assumed, not earned (Web Worker Daily, 2006). Team members, supervisors, leaders, and managers must create and support a culture that encourages openness, trust, and respect. This will provide a foundation upon which all elements of team effectiveness can be built and supported (Nydegger, 2009).

In summary, to be successful, virtual teams must have appropriate:

- $\quad$ Technology
- $\quad$ HR policies
- $\quad$ Training and development for team leaders and members
- $\quad$ Appropriate organizational culture
- $\quad$ Leadective leadership
Leader/member competency (Duarte \& Snyder, 1999)

Organizations that are prepared and willing to enter into the virtual world will have opportunities that will help them succeed and prosper. How they meet these opportunities and challenges will determine how well they achieve their goals. 


\section{REFERENCES}

1. Ahuja, M. K., Galletta, D. F., \& Carley, K. M. (2003). Individual centrality and performance in virtual R\&D groups: An empirical study. Management Science, 49(1), 21-38.

2. Bjørn, P., \& Ngwenyama, O. (2009). Virtual team collaboration: Building shared meaning, resolving breakdowns and creating translucence. Information Systems Journal, 19, 227-253.

3. Boiney, L. G. (2001). Gender impacts virtual work teams. Graziadio Business Report, 4(4), 5.

4. Box, N., Olson, J., Gergle, D., Olson, G., \& Wright, Z. (2002). Effects of four computer-mediated communications channels on trust development. In Proc. SIGCHI.

5. Cascio, W. F. (1999). Virtual workplaces: Implications for organizational behavior. In C. L. Cooper \& D. M. Rousseau (Eds.), Trends in organizational behavior, Vol. 6: The virtual organization. (pp. 1-14). New York, NY US: John Wiley \& Sons Ltd.

6. Cascio, W. F. (2000). Managing a Virtual Workplace. The Academy of Management Executive (1993), 14(3), 81-90.

7. Daily, W. W. (2006). Seven habits of successful virtual teams. Retrieved November 24, 2008, from http://webworkerdaily.com/2006/12/17/seven-habits-of-successful-virtual-teams/

8. Dean, B. (1998). The psychologist as a virtual coach. The Independent Practitioner, 18(4), 188-189.

9. Duarte, D. L., \& Snyder, N. T. (1999). Mastering Virtual Teams. San Francisco, CA: Jossey-Bass.

10. Dubé, L., \& Robey, D. (2008). Surviving the paradoxes of virtual teamwork. Information Systems Journal, 19, 3-30.

11. Finholt, T., \& Sproull, L. S. (1990). Electronic groups at work. Organization Science, 1(1), 41-64.

12. Goodman, P. S., Ravlin, E., \& Schminke, M. (1987). Understanding groups in organizations. Research in Organizational Behavior, 9, 121-173.

13. Gould, D. (1997). Leading virtual teams. Virtual Organization Retrieved November 27, 2008, from http://www.seanet.com/ daveg/ltv.htm

14. Gould, D. (2006). Fifth generation work. Virtual Organization Retrieved November 20, 2008, from http://www.seanet.com/ daveg/vrteams.htm

15. Handy, C. (1995). Trust and the Virtual Organization. Harvard Business Review, 73(3), 40-50.

16. Hertel, G., Geister, S., Konradt, U. (2005). Managing virtual teams: A review of current empirical research. Human Resource Management Review, 15, 69-95.

17. Jarvenpaa, S. L., \& Leidner, D. E. (1999). Communication and trust in global virtual teams. Organization Science, 10(6), 791-815.

18. Kimball, L. (1997). Managing virtual teams. Paper presented at the Teams Strategies Conference, Toronto, Canada.

19. Lea, M., \& Spears, R. (1991). Computer-mediated communication, de-individuation and group decisionmaking. International Journal of Man-Machine Studies, 34(2), 283-301.

20. Lind, M. R. (1999). The gender impact of temporary virtual work groups. IEEE Transactions of Professional Communication, 42(4), 10.

21. McKenna, K. Y. A. (2008). Influences on the nature and functioning of online groups. In A. Barak (Ed.), Psychological Aspects of Cyberspace: Theory, Research, Applications. (pp. 228-242). New York, NY: Cambridge University Press.

22. McKenna, K. Y. A., \& Green, A. S. (2002). Virtual group dynamics. Group Dynamics: Theory, Research, and Practice, 6(1), 116-127.

23. McLeod, P. L. (1992). An assessment of the experimental literature on electronic support of group work: Results of a meta-analysis. Human-Computer Interaction, 7(3), 257-280.

24. Merriman, K. K., Schmidt, S. M., \& Dunlap-Hinkler, D. (2007). Profiling virtual employees: The impact of managing virtually. Journal of Leadership \& Organizational Studies, 14, 6-15.

25. Nardi, B., \& Whittiker, S. (2002). The place of face to face communication in distributed work. In P. Hinds \& S. Kiesler (Eds.), Distributed Work: New Research on Working Across Distance Using Technology (pp. 83-110). Cambridge, MA: MIT Press.

26. Oakley, J. G. (1998). Leadership processes in virtual teams and organizations. The Journal of Leadership Studies, 5, 3-15.

27. Smith, J. Y., \& Vanecek, M. T. (1990). Dispersed Group Decision Making Using Nonsimultaneous Computer Conferencing: A Report of Research. Journal of Management Information Systems, 7(2), 71-92. 
28. Thomas, W. I. (1923). The Unadjusted Girl. Boston: Little, Brown, \& Co.

29. Toffler, A. (1970). Future Shock. New York: Random House.

30. Tuckman, B. T. (1965). Developmental sequence in small groups. Psychological Bulletin, 63, 384-399.

31. Walther, J. B. (1992). Interpersonal effects in computer-mediated interaction: A relational perspective. Communication Research, 19(1), 52-90.

32. Walther, J. B., Bunz, U., \& Bazarova, N. N. (2005). The rules of virtual groups. Paper presented at the 38th Hawaii International Conference on System Science, Hawaii.

33. Weisband, S., \& Atwater, L. (1999). Evaluating self and others in electronic and face-to-face groups. Journal of Applied Psychology, 84(4), 632-639.

34. Wiesenfeld, B. M., Raghuram, S., \& Garud, R. (1999). Managers in a virtual context: The experience of self-threat and its effects on virtual work organizations. In C. L. Cooper \& D. M. Rousseau (Eds.), Trends in organizational behavior, Vol. 6: The virtual organization. (pp. 31-44). New York, NY US: John Wiley \& Sons Ltd. 